Portland State University

PDXScholar

\title{
Addressing Homeless Encampments on Public Right-of-Way: A Knowledge Transfer Project
}

Andrée Tremoulet

Portland State University, andree@commonworksconsulting.com

Follow this and additional works at: https://pdxscholar.library.pdx.edu/usp_fac

Part of the Urban Studies and Planning Commons

Let us know how access to this document benefits you.

\section{Citation Details}

Tremoulet, A., Addressing Homeless Encampments on Public Right-of-Way: A Knowledge Transfer Project. OTREC-SS-731. Portland, OR: Transportation Research and Education Center (TREC), 2013. http://dx.doi.org/10.15760/trec.137

This Report is brought to you for free and open access. It has been accepted for inclusion in Urban Studies and Planning Faculty Publications and Presentations by an authorized administrator of PDXScholar. Please contact us if we can make this document more accessible: pdxscholar@pdx.edu. 


\title{
S)OTREC
}

FINAL REPORT

Addressing Homeless Encampments on Public Right-Of-Way:

A Knowledge Transfer Project

\author{
OTREC-SS-73 1 \\ September 2013
}





\title{
ADDRESSING HOMELESS ENCAMPMENTS ON PUBLIC RIGHT-OF-WAY A KNOWLEDGE TRANSFER PROJECT
}

\section{Final Report}

\section{OTREC}

\author{
by
}

Andrée Tremoulet, Ph.D.

Portland State University

for

Oregon Transportation Research and Education Consortium (OTREC)

P.O. Box 751

Portland, OR 97207

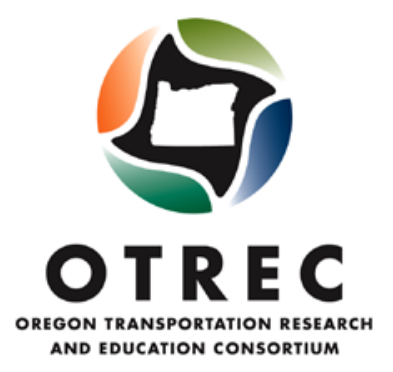

September 2013 



\begin{tabular}{|c|c|c|c|c|}
\hline \multicolumn{5}{|c|}{ Technical Report Documentation Page } \\
\hline 1. Report No. & \multicolumn{2}{|l|}{ 2. Government Accession No. } & \multicolumn{2}{|c|}{ 3. Recipient's Catalog No. } \\
\hline \multicolumn{3}{|c|}{$\begin{array}{l}\text { 4. Title and Subtitle } \\
\text { Addressing Homeless Encampments on Public Right-of-Way: A Knowledge Transfer Project }\end{array}$} & \multicolumn{2}{|c|}{$\begin{array}{l}\text { 5. Report Date } \\
\text { September 30, } 2013\end{array}$} \\
\hline \multicolumn{3}{|c|}{ Addressing Homeless Encampments on Public Right-of-Way: A Knowledge Transfer Project } & \multicolumn{2}{|c|}{ 6. Performing Organization Code } \\
\hline $\begin{array}{l}\text { 7. Author(s) } \\
\text { Andrée Tremoulet, Ph.D. }\end{array}$ & & \multicolumn{2}{|c|}{ 8. Performing Organization Report No. } \\
\hline \multirow{2}{*}{\multicolumn{4}{|c|}{$\begin{array}{l}\text { Center for Urban Studies } \\
\text { Portland State University } \\
\text { PO Box } 751 \\
\text { Portland, OR } 97207\end{array}$}} & 10. Work Unit No. (TRAIS) \\
\hline & & & \multicolumn{2}{|c|}{$\begin{array}{l}\text { 11. Contract or Grant No. } \\
\text { 2013-641 }\end{array}$} \\
\hline \multirow{2}{*}{\multicolumn{3}{|c|}{$\begin{array}{l}\text { 12. Sponsoring Agency Name and Address } \\
\text { Oregon Transportation Research } \\
\text { and Education Consortium (OTREC) } \\
\text { P.O. Box } 751 \\
\text { Portland, Oregon } 97207\end{array}$}} & \multicolumn{2}{|c|}{$\begin{array}{l}\text { 13. Type of Report and Period Covered } \\
\text { Final } 3 / 1 / 2013-9 / 30 / 2013\end{array}$} \\
\hline & & & \multicolumn{2}{|c|}{ 14. Sponsoring Agency Code } \\
\hline \multicolumn{5}{|l|}{ 15. Supplementary Notes } \\
\hline \multicolumn{5}{|c|}{$\begin{array}{l}\text { This technology transfer project provided practical information about how to address homeless encampments on public right-of-way to } \\
\text { transportation professionals, including Federal Highway Administration officials, Oregon public works directors, transportation educators and } \\
\text { students. }\end{array}$} \\
\hline \multicolumn{5}{|c|}{$\begin{array}{l}\text { Through a previous project funded by OTREC and Oregon Department of Transportation, research had been conducted about the extent of the } \\
\text { issue among state transportation agencies and how they were addressing it. The prior grant also provided for the development of a Best } \\
\text { Practices Guide. The current project enabled staff to present the results of the research to transportation practitioners and policy analysts } \\
\text { through multiple forums and media coverage. }\end{array}$} \\
\hline 17. Key Words & & $\begin{array}{l}\text { 18. D } \\
\text { No } \\
\text { wh }\end{array}$ & $\begin{array}{l}\text { bution Statement } \\
\text { ictions. Copies ave } \\
\text { rec.us }\end{array}$ & EE: \\
\hline $\begin{array}{l}\text { 19. Security Classification (of this report) } \\
\text { Unclassified }\end{array}$ & $\begin{array}{l}\text { 20. Security Classification (of } \\
\text { Unclassified }\end{array}$ & page) & $\begin{array}{l}\text { 21. No. of Pages } \\
25\end{array}$ & 22. Price \\
\hline
\end{tabular}




\section{ACKNOWLEDGEMENTS}

This project was funded by the Oregon Transportation Research and Education Consortium (OTREC).

\section{DISCLAIMER}

The contents of this report reflect the views of the authors, who are solely responsible for the facts and the accuracy of the material and information presented herein. This document is disseminated under the sponsorship of the U.S. Department of Transportation University Transportation Centers Program in the interest of information exchange. The U.S. Government assumes no liability for the contents or use thereof. The contents do not necessarily reflect the official views of the U.S. Government. This report does not constitute a standard, specification, or regulation. 


\section{TABLE OF CONTENTS}

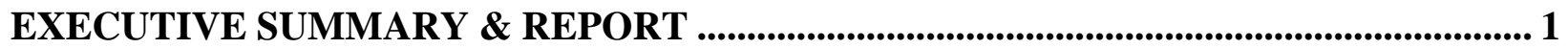

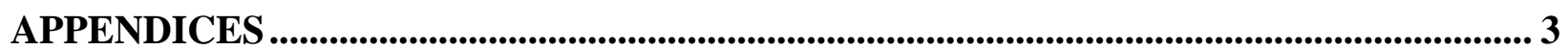

APPENDIX A: STRATEGIES FOR ADDRESSING THE CHALLENGES OF TRANSIENT

POPULATIONS ON TRANSPORTATION FACILITIES WEBINAR............................. 3

APPENDIX B: TECHNICAL PROGRAM …………………………………………........ 15

APPENDIX C: HIDING IN PLAIN SIGHT: BALDOCK RESTORATION PROJECT......... 16

APPENDIX D: RESPONSE TO 2013 CALL FOR TRANSPORTATION AND

ENVIRONMENTAL RESEARCH IDEAS ………………………………………..... 22 


\section{EXCUTIVE SUMMARY \& REPORT}

This technology transfer project provided practical information about how to address homeless encampments on public right-of-way to transportation professionals, including Federal Highway Administration officials, Oregon public works directors, transportation educators and students.

Through a previous project funded by OTREC and Oregon Department of Transportation, research had been conducted about the extent of the issue among state transportation agencies and how they were addressing it. The prior grant also provided for the development of a Best Practices Guide. The current project enabled staff to present the results of the research to transportation practitioners and policy analysts through multiple forums and media coverage.

Specific activities, outcomes and results of the current project include the following:

- Webinar for FHWA staff nationally and others. The two-hour webinar, hosted by FHWA's Office of Human Environment was conducted on May 23 ${ }^{\text {rd }}$, 2013. According to the report provided by FHWA, 161 individuals registered and 141 participated. Staff from 16 FHWA Divisions, 13 state DOTs, local jurisdictions, agencies and nonprofit organizations participated. A copy of the FHWA-prepared report appears as Appendix A. The archived webinar can be found here: https://connectdot.connectsolutions.com/p6jemor31o7/

- Presentation to the Oregon Chapter of the American Public Works Association Conference. A one-hour interactive presentation was provided to an audience of approximately 25 Oregon public works officials on Thursday, April $4^{\text {th }}$, at the 2013 Spring Conference of the Oregon Chapter of the American Public Works Association. The session included a presentation and dialogue with the attendees. Several participants stayed after the session to talk further, and one participant contacted us after the conference to let us know that our presentation and guidebook had been helpful in developing a county policy on the issue. A PDF of the Agenda appears as Appendix B.

- Presentation at PSU Transportation Seminar. A 40-minute presentation was made at the weekly PSU Transportation Seminar on March $15^{\text {th }}$. The event was sparsely attended (perhaps 10 participants); it was the last Friday of the winter term. A brief question and answer session followed the presentation.

- Additional Contacts.

0 A report on the FHWA Webinar was presented by staff representing the Department of Transportation at a meeting of the United States Interagency Committee on Homelessness (USICH), attended by cabinet-level officials and/or their appointees.

o We responded to requests for information from a TxDOT staff person from Austin, Texas and a researcher from Mexico.

- Metroscape Article. A manuscript was prepared for the summer 2013 issue of Metroscape. However, as the remaining articles in the issue focused on urban chickens, we asked that the manuscript be held for a later issue. A copy of the manuscript appears as Appendix C. 
Further activities (beyond those in the original scope of work) that were completed during this grant period include:

- Media Coverage. The research for this project was featured in an article (suggested by OTREC staff) in Atlantic Cities. It can be found here: http://www.theatlanticcities.com/housing/2013/02/why-homelessness-transportationissue/4577/

- Submission of Proposed Research Topic. A transportation research idea, developed in collaboration with the co-chair of the TRB Environmental Justice Subcommittee and FHWA, was submitted in response to a Call for Transportation and Environmental Research Ideas. A copy of the submission appears as Appendix D.

- Future TRB Annual Meeting Crosscutting Topics Session: A proposal for a crosscutting session on homeless encampments has been submitted by the chairs of the Environmental Justice and Property Maintenance Subcommittees for the January 2014 TRB Annual Meeting. We are awaiting information about the outcome of that proposal.

Future activities that may occur beyond the duration of this grant include:

- Manuscript Preparation: A manuscript to submit to an academic journal is underway but will not be complete by the end of this grant period. Goal is to complete draft manuscript by December 15, 2013.

- Further Presentations: Homelessness on public right-of-way continues to be a relevant public policy topic. In Portland, the change of administration has resulted in a shift in policy regarding urban campgrounds on public land, including transportation land in the Lents neighborhood. As word continues to get out about this project, additional speaking requests are anticipated. For example, we have been asked to present at the monthly meeting of the multi-agency homeless committee in Washington County on October 2, 2013. 


\section{APPENDIX A}

FHWA Emerging Issue: Strategies for Addressing the Challenges of Transient Populations on Transportation Facilities Webinar

\section{Summary}

The Federal Highway Administration's (FHWA) Office of Human Environment (HEPH) hosted a webinar to address homeless encampments on transportation facilities on May 23, 2013, from 1:00 to 3:00 p.m., Eastern Time.

The webinar's speakers included:

- Sharlene Reed, Community Planner, Office of Human Environment, FHWA

- Bruce Bradley, Realty Specialist, Office of Real Estate Services, FHWA

- Mary Jane Daluge, Realty Specialist, Office of Real Estate Services, FHWA

- Ellen M. Bassett, Ph.D., Associate Professor, Dept. of Urban and Environmental Planning

University of Virginia

- Andrée Tremoulet, Ph.D., Research Associate, Center for Urban Studies-CUS Portland State University A recording of the webinar is available at https://connectdot.connectsolutions.com/p6jemor31o7/

One hundred and sixty-one (161) individuals registered for the webinar and one-hundred and forty-one (141) participated. Questions were asked on a variety of related issues and participants' understanding was measured through polling questions.

This report provides a detailed summary of the FHWA webinar on Strategies for Addressing the Challenges of Transient Populations on Transportation Facilities.

\section{Promotion}

The webinar was promoted through NHI, the Loop, an e-mail announcement from the HEPH Office Director and several emails to external organizations.

\section{Registration}

The individuals who participated in the session represented sixteen (16) FHWA Divisions (AZ, CA, CO, FL,GA, MO, MS, MT, NM, NV,OK, PA, SC, TX, WA, WV) thirteen (13) State DOTS (AK, AZ, FL, GA, HI, MI, MT, NV,OK, TX,VA, WA,WY), FHWA Headquarters, local officials, municipalities and external organizations such as Clackamas County Social Services, the Nature Conservancy, Hernando County MPO, Baton Rouge Bike Club, WalkBoston and Sequoia Mental Health Services.

A full list of registrants begins on page 6 .

\section{Participation}

Of the 161 individuals registered, 87\% (141 individuals) participated in the webinar. More than $73 \%$ of these participants stayed on the webinar until the ending time 3:00 p.m. This number does not include the speakers and the FHWA staff members in the conference room. During the webinar, participants were encouraged to submit questions via a chat pod. Five (5) questions on 
the content of the presentations were asked in the chat. Additional questions were addressed over the phone.

The topics of these questions include:

- Do you have any examples of commitment in a NEPA document to work with homeless within a project site as part of project mitigation?

- Does Title VI have a role in situations of homeless on public lands?

- Who should take the lead in providing education and outreach on this issue?

\section{Polling Questions}

Five polling questions were asked during the webinar.

\section{Question 1}

Which choice below best describes your field of work?

\begin{tabular}{|l|l|c|}
\hline Answer & Number & Percent \\
\hline Transportation & 48 & $72.7 \%$ \\
\hline $\begin{array}{l}\text { Other public } \\
\text { works }\end{array}$ & 1 & $1.5 \%$ \\
\hline Social services & 3 & $4.5 \%$ \\
\hline $\begin{array}{l}\text { Community } \\
\text { Justice }\end{array}$ & 8 & $12.1 \%$ \\
\hline Planning & 6 & $9.1 \%$ \\
\hline Other & 0 & 0 \\
\hline Total & $\mathbf{6 6}$ & $\mathbf{1 0 0} \%$ \\
\hline
\end{tabular}

\section{Question 2}

Which choice below best describes your professional role?

\begin{tabular}{|l|l|c|}
\hline Answer & Number & Percent \\
\hline $\begin{array}{l}\text { Manager or } \\
\text { supervisor }\end{array}$ & 27 & $41.5 \%$ \\
\hline $\begin{array}{l}\text { Planner or } \\
\text { policy analyst }\end{array}$ & 16 & $24.6 \%$ \\
\hline Elected official & 1 & $1.5 \%$ \\
\hline Consultant & 2 & $3.1 \%$ \\
\hline $\begin{array}{l}\text { Direct service } \\
\text { provider }\end{array}$ & 1 & $1.5 \%$ \\
\hline Advocate & 1 & $1.5 \%$ \\
\hline Researcher & 2 & $3.1 \%$ \\
\hline Attorney & 15 & $23.1 \%$ \\
\hline Other & 0 & 0 \\
\hline Total & $\mathbf{6 5}$ & $100 \%$ \\
\hline
\end{tabular}




\section{Question 3}

Why are you interested in this topic? (please choose all that apply)

\begin{tabular}{|l|l|l|}
\hline Answer & Number & Percent \\
\hline $\begin{array}{l}\text { My agency/ } \\
\text { business } \\
\text { encounters } \\
\text { homeless } \\
\text { encampments } \\
\text { on public land } \\
\text { on a regular } \\
\text { basis }\end{array}$ & 25 & $47.1 \%$ \\
\hline $\begin{array}{l}\text { This is a new } \\
\text { topic that } \\
\text { interests me }\end{array}$ & 15 & $28.3 \%$ \\
\hline $\begin{array}{l}\text { I am looking for } \\
\text { solutions to this } \\
\text { problem }\end{array}$ & 7 & $13.2 \%$ \\
\hline $\begin{array}{l}\text { I don't know } \\
\text { where to turn to } \\
\text { get help with } \\
\text { this issue }\end{array}$ & 1 & $1.8 \%$ \\
\hline Other & 5 & $9.4 \%$ \\
\hline Total & 53 & $100 \%$ \\
\hline
\end{tabular}

\section{Question 4}

What additional steps do you think should be initiated at the national level to better address this issue? (check all that apply)

\begin{tabular}{|l|l|l|}
\hline Answer & Number & Percent \\
\hline $\begin{array}{l}\text { Additional research about the scope } \\
\text { and nature of the problem }\end{array}$ & 10 & $16.6 \%$ \\
\hline $\begin{array}{l}\text { More detailed resources (website, } \\
\text { etc.) with links to ways to address } \\
\text { this issue }\end{array}$ & 15 & $25 \%$ \\
\hline $\begin{array}{l}\text { Interagency (HUD, DOT) } \\
\text { collaboration to develop proactive } \\
\text { solutions that can be adopted locally }\end{array}$ & 16 & $26.6 \%$ \\
\hline $\begin{array}{l}\text { Research and guidance on how to } \\
\text { address impacts on homeless } \\
\text { populations in the design and } \\
\text { construction of projects }\end{array}$ & 16 & $26.6 \%$ \\
\hline Other & 3 & $5 \%$ \\
\hline Total & $\mathbf{6 0}$ & $100 \%$ \\
\hline
\end{tabular}




\section{Question 5}

What steps might you take as a result of participating in this webinar? (Check all that apply)

\begin{tabular}{|l|l|c|}
\hline Answer & Number & Percent \\
\hline $\begin{array}{l}\text { Share information (and } \\
\text { link to webinar/Best } \\
\text { Practices Guide) with } \\
\text { others in my } \\
\text { office/field }\end{array}$ & 6 & $31.5 \%$ \\
\hline $\begin{array}{l}\text { Begin considering how } \\
\text { to collaborate with } \\
\text { professionals from } \\
\text { other fields to address } \\
\text { some issues we have } \\
\text { locally }\end{array}$ & 6 & $31.5 \%$ \\
\hline $\begin{array}{l}\text { Suggest we have a } \\
\text { speaker who can } \\
\text { address this topic at our } \\
\text { next professional } \\
\text { association conference }\end{array}$ & 3 & $15.7 \%$ \\
\hline $\begin{array}{l}\text { Consider developing a } \\
\text { plan for my own } \\
\text { agency }\end{array}$ & 3 & $15.7 \%$ \\
\hline Other & 1 & $100 \%$ \\
\hline Total & $\mathbf{1 9}$ & \\
\hline
\end{tabular}

\section{Participant Feedback}

Twenty-five (25) participants completed an evaluation at the conclusion of the webinar. Participants were asked questions on the quality of the webinar content, its structure, the accessibility of the information and its relevance to their work. Highlights of the evaluation include:

- $88 \%$ of respondents agreed that the webinar "positively impacted their understanding of the topic."

- $70 \%$ of respondents agreed that their "expectations for the webinar were effectively met."

- $87 \%$ stated that the webinar's "topic and/or agenda was relevant to my success on the job."

The webinar evaluation offered an opportunity for participants to submit comments regarding the content and structure of the webinar. Nine (9) participants offered comments for this webinar. Highlights are listed below:

- Thank you for this presentation. It was interesting on the approaches used to help resolve problems to effectively start and work on a process to address the homelessness problem which can be very personal to the people living in the community. 
- Thank you looking at this topic -- treating homeless people as "trespassers" doesn't help society in the long run. Using law enforcement to clear a project site without also having social services assistance available at the site is inhumane. The majority of homeless people do not choose to be homeless.

- An additional thought to consider when dealing with local partners. The issue of homelessness in many communities is far greater than the resources available to treat them. As such, some believe that these established homeless encampments, although not ideal, represent a viable solution to the problem and should be supported.

- Nicely done.

\section{Recommendations}

Based on this webinar, the following recommendations should be considered to enhance and improve future FHWA research program webinars:

- Consider offering multiple sessions of the same webinar. This webinar attracted an engaged group of participants, who in some cases received less than a week's notice. Offering the session in the future could help to attract a wider audience.

- Invite outside experts to field questions and participate in the discussion. Having a broader group of experts allows for an exchange of ideas and information sharing rather than just pushing out information.

\section{$\underline{\text { List of Registrants }}$}

\begin{tabular}{|l|l|l|l|}
\hline Abbott, Sarah & & sabbott@clackamas.us & Clackamas County \\
\hline Aberle, Barbara & 3609052186 & aberleb@wsdot.wa.gov & $\begin{array}{l}\text { Washington Department of } \\
\text { Transportation }\end{array}$ \\
\hline Alcorn, Caitlin & 8505532227 & caitlin.alcorn@dot.gov & FHWA Florida Division \\
\hline $\begin{array}{l}\text { ALDERTON, } \\
\text { JULIE }\end{array}$ & 5177021821 & julie.alderton@dot.gov & USD0T-FHWA \\
\hline ALLEN, KATY & $\begin{array}{l}404-562- \\
3657\end{array}$ & katy.allen@dot.gov & FHWA GEORGIA DIVISION \\
\hline $\begin{array}{l}\text { Anderson, } \\
\text { Kimberly }\end{array}$ & kimberly.anderson@dot.gov & FHWA \\
\hline Anderson, Linda & $\begin{array}{l}850-553- \\
2226\end{array}$ & linda.anderson@dot.gov & FHWA \\
\hline Anderson, Marc & & marcus.anderson@wyo.gov & WYDOT \\
\hline $\begin{array}{l}\text { Aronson, } \\
\text { Matthew }\end{array}$ & 2024023554 & matthew.k.aronson@hud.gov & $\begin{array}{l}\text { US Department of Housing and } \\
\text { Urban Development }\end{array}$ \\
\hline banks, teresa & $\begin{array}{l}404-562- \\
3592\end{array}$ & teresa.banks@dot.gov & fhwa-rc \\
\hline Banta, Kari & 512 217 9527 & kari.banta@sierraclub.org & Sierra Club \\
\hline Barlow, Diana & $(405) 521-$ & dbarlow@odot.org & Oklahoma Department of \\
\hline
\end{tabular}




\begin{tabular}{|l|l|l|l|}
\hline & 2648 & & Transpotation \\
\hline Beeson, Ethan & $\begin{array}{l}713-802- \\
5471\end{array}$ & ethan.beeson@txdot.gov & TxDOT \\
\hline $\begin{array}{l}\text { Benton-Hooks, } \\
\text { Carla }\end{array}$ & $\begin{array}{l}404-631- \\
1415\end{array}$ & cbenton-hooks@dot.ga.gov & GDOT \\
\hline Betlyon, Brian & & brian.betlyon@dot.gov & FHWA RC \\
\hline Black, Jason & $\begin{array}{l}206-515- \\
3974\end{array}$ & blackj@wsdot.wa.gov & WSDOT-Ferries Division \\
\hline Blount, Wesley & $\begin{array}{l}202-366- \\
0799\end{array}$ & wesley.blount@dot.gov & FHWA \\
\hline $\begin{array}{l}\text { Borcherding, } \\
\text { Hillary }\end{array}$ & hborcherding@walkboston.org & WalkBoston \\
\hline Bowers, Fred & $\begin{array}{l}202-366- \\
2374\end{array}$ & frederick.bowers@dot.gov & FHWA \\
\hline $\begin{array}{l}\text { Bowman, R. } \\
\text { Larry }\end{array}$ & $\begin{array}{l}404-631- \\
1362\end{array}$ & lbowman@dot.ga.gov & $\begin{array}{l}\text { Georgia Department of } \\
\text { Transportation (GDOT) }\end{array}$ \\
\hline
\end{tabular}

\begin{tabular}{|l|l|l|l|}
\hline Branton, Tanya & $850-330-1599$ & tanya.branton@dot.state.fl.us & FDOT \\
\hline Breck, Andrew & $617-494-2213$ & andrew.breck@dot.gov & DOT/Volpe Center \\
\hline Brown, Ann & $503-986-2122$ & Ann.Brown@hcs.state.or.us & $\begin{array}{l}\text { Oregon Housing and } \\
\text { Community Services }\end{array}$ \\
\hline Brown, Carr & $601-965-7326$ & Carr.Brown@dot.gov & FHWA - MS Division \\
\hline burk, brian & 5129740899 & brian.burk@txdot.gov & txdot \\
\hline Cardona, Aldo & $915-790-4482$ & aldo.cardona@txdot.gov & TXDOT \\
\hline Clark, Chris & & percist@gmail.com & Baton Rouge Bike Club \\
\hline Clark, Laura & $407-971-8850$ & lclark@inwoodinc.com & Inwood Consulting Engineers \\
\hline Clotfelter, Don & $360-357-2614$ & clotfed@wsdot.wa.gov & WSDOT \\
\hline Colson, Regina & & regina.colson@dot.state.fl.us & FDOT \\
\hline $\begin{array}{l}\text { conard, } \\
\text { summer }\end{array}$ & & sconard@newhopehousing.org & new hope housing \\
\hline Cook, Steve & $517-204-3099$ & cooks9@michigan.gov & Michigan DOT \\
\hline $\begin{array}{l}\text { Copeland, } \\
\text { Kathryn }\end{array}$ & $803-737-4477$ & copelandke@scdot.org & SCDOT \\
\hline Cubic, Aaron & $541-450-6000$ & acubic@grantspassoregon.gov & City of Grants Pass \\
\hline Curry, Melanie & 5108458865 & curryme@cal.berkeley.edu & SFCTA \\
\hline Davila, Lisa & $503-726-3816$ & ldavila@luke-dorf.org & Luke-Dorf \\
\hline Davis, Teri & $\begin{array}{l}\text { (806) 775- } \\
1676\end{array}$ & tbdavis@mylubbock.us & $\begin{array}{l}\text { Lubbock Metropolitan Planning } \\
\text { Organization }\end{array}$ \\
\hline Day, Amanda & & aday@daycommunications.com & Best Foot Forward \\
\hline
\end{tabular}




\begin{tabular}{|c|c|c|}
\hline Diehl, Sean & sdiehl@dot.ga.gov & GDOT \\
\hline Dix, Dennis & dennisd@hernandocounty.us & Hernando County MPO \\
\hline
\end{tabular}

\begin{tabular}{|l|l|l|l|}
\hline dixon, marc & & marc.dixon@dot.gov & fhwa \\
\hline Drost, Dale & $\begin{array}{l}602-245- \\
0241\end{array}$ & ddrost@azdot.gov & ADOT \\
\hline $\begin{array}{l}\text { dumbuya, } \\
\text { mohamed }\end{array}$ & 8047753339 & mohamed.dumbuya@dot.gov & fhwa \\
\hline $\begin{array}{l}\text { Duncan- } \\
\text { Augustt, } \\
\text { Maggie }\end{array}$ & $\begin{array}{l}202-366- \\
9901\end{array}$ & maggie.duncan-augustt@dot.gov & FHWA \\
\hline $\begin{array}{l}\text { Edwards, } \\
\text { Andrew }\end{array}$ & andrew.edwards@dot.gov & FHWA GA Division \\
\hline $\begin{array}{l}\text { Enderle, } \\
\text { Evelyn }\end{array}$ & $\begin{array}{l}720-963- \\
3002\end{array}$ & evelyn.enderle@dot.gov & FHWA - Colorado Division \\
\hline Eubanks, Janet & $\begin{array}{l}202-912- \\
7149\end{array}$ & jeubanks@blm.gov & DOI, BLM \\
\hline $\begin{array}{l}\text { Evans, } \\
\text { Annette }\end{array}$ & $\begin{array}{l}503-846- \\
4760\end{array}$ & Annette_Evans@co.washington.or.us & $\begin{array}{l}\text { Washington County } \\
\text { Separtment of Housing }\end{array}$ \\
\hline Fauver, Kirk & $\begin{array}{l}512-536- \\
5952\end{array}$ & kirk.fauver@dot.gov & FHWA Texas Division \\
\hline $\begin{array}{l}\text { Fedora, } \\
\text { Richard }\end{array}$ & r.wayne.fedora@dot.gov & FHWA \\
\hline Fisher, Linda & $\begin{array}{l}503-655- \\
8492\end{array}$ & lindafis@clackamas.us & $\begin{array}{l}\text { Clackamas County Social } \\
\text { Services }\end{array}$ \\
\hline Fordjour, Kojo & & fordjok@wsdot.wa.gov & WSF \\
\hline Frye, Mary & & Mary.Frye@dot.gov & FHWA \\
\hline Gardner, Erin & gardnee@wsdot.wa.gov & WSDOT \\
\hline Gerwein, Joel & jgerwein@scc.ca.gov & Falifornia State Coastal \\
\hline $\begin{array}{l}\text { Givan, } \\
\text { Shundreka }\end{array}$ & $\begin{array}{l}720-963- \\
\text { Gomez, } \\
\text { Patrick }\end{array}$ & patrick.gomez@dot.gov & FHWA \\
\hline $\begin{array}{l}\text { Gottlieb, } \\
\text { Joyce }\end{array}$ & joyce.gottlieb@dot.gov & FHWA-HCR \\
\hline Green, Donna & donna.green@dot.state.fl.us & $\begin{array}{l}\text { Transportation Public } \\
\text { Information Office }\end{array}$ \\
\hline
\end{tabular}




\begin{tabular}{|c|c|c|c|}
\hline \begin{tabular}{|l|l} 
Grimm, Lewis & 7 \\
6
\end{tabular} & \begin{tabular}{|l}
$703-404-$ \\
6289
\end{tabular} & lewis.grimm@dot.gov & \begin{tabular}{|l} 
FHWA, Eastern Federal \\
Lands Highway Division
\end{tabular} \\
\hline \begin{tabular}{|l} 
Gurganus, \\
Stephen
\end{tabular} & $\begin{array}{l}919-515- \\
9351\end{array}$ & sjgurgan@ncsu.edu & CTE/ITRE/NCSU \\
\hline Hall, Faith & & faith.hall@dot.gov & DOT \\
\hline $\begin{array}{l}\text { Hasselbach, } \\
\text { Brian }\end{array}$ & & Brian.Hasselbach@dot.gov & FHWA - Montana Division \\
\hline Hayes, Janet & \begin{tabular}{|l}
$404-562-$ \\
3579
\end{tabular} & janet.hayes@dot.gov & FHWA, Resource Center \\
\hline Haynes, Angelo & $\begin{array}{l}(805) 258- \\
7212\end{array}$ & ahaynes@tnc.org & The Nature Conservancy \\
\hline Heitmann, Greg & $g \mid \begin{array}{l}505-820- \\
2027\end{array}$ & greg.heitmann@dot.gov & FHWA - NM DIV \\
\hline Hekter, Jessica & & jessica.hekter@dot.gov & FHWA-SC \\
\hline Helgath, Sheila & $\begin{array}{l}206-515- \\
3911\end{array}$ & helgats@wsdot.wa.gov & Washington State Ferries \\
\hline Henslee, Dave & $\begin{array}{l}(541) 766- \\
6778 \\
\end{array}$ & dave.henslee@corvallisoregon.gov & Corvallis Police Department \\
\hline Herrera, Corina & $\begin{array}{l}(804) 786- \\
2730\end{array}$ & corina.herrera@vdot.virginia.gov & $\begin{array}{l}\text { Virginia Department of } \\
\text { Transportation }\end{array}$ \\
\hline HIrt, Debbie & & Deborah.Hirt@dot.state.nj.us & $\begin{array}{l}\text { NJ Dept. of Transportation - } \\
\text { Office of Community Relations }\end{array}$ \\
\hline Hodge, Sandra & \begin{tabular}{|l|}
$305-445-$ \\
2900
\end{tabular} & sandra.hodge@stantec.com & Stantec Consulting Services \\
\hline Hoffman, Barry & $\begin{array}{l}541-917- \\
7606\end{array}$ & barry.hoffman@cityofalbany.net & City of Albany, Oregon \\
\hline $\begin{array}{l}\text { Hoffman, } \\
\text { Elizabeth }\end{array}$ & & elizabeth.hoffman@dot.gov & FHWA \\
\hline $\begin{array}{l}\text { Hughes, } \\
\text { Andrew }\end{array}$ & $\begin{array}{l}907-465- \\
1776\end{array}$ & andy.hughes@alaska.gov & $\begin{array}{l}\text { Alaska Department of } \\
\text { Transportation and Public } \\
\text { Facilities }\end{array}$ \\
\hline Jackson, Sandra & & Sandra.Jackson@dot.gov & FHWA-DC \\
\hline \begin{tabular}{|l|} 
Jacobs-Brown, \\
Michelle
\end{tabular} & $\begin{array}{l}360-905- \\
2161\end{array}$ & jacobsm@wsdot.wa.gov & WSDOT \\
\hline James, Carolyn & \begin{tabular}{|l} 
202-493- \\
0353
\end{tabular} & carolyn.james@dot.gov & FHWA \\
\hline James, Denesha & $\begin{array}{l}\begin{array}{l}301-848- \\
2893\end{array} \\
\end{array}$ & denesha.james@dot.gov & FHWA \\
\hline Jones, John & \begin{tabular}{|l}
$202-493-$ \\
0350
\end{tabular} & john.m.jones@dot.gov & $\begin{array}{l}\text { FHWA, Office of Real Estate } \\
\text { Services }\end{array}$ \\
\hline
\end{tabular}




\begin{tabular}{|l|l|l|l|}
\hline Jones, Rosemary & & Rosemary.jones@dot.gov & FHWA \\
\hline Kerns, Will & 3038154264 & wkerns@openplan.net & $\begin{array}{l}\text { Open Plan Consultants } \\
\text { LLC }\end{array}$ \\
\hline Koester, Rudy & 515.239 .5245 & rkoester@city.ames.ia.us & Ames Area MPO \\
\hline Kord, Nicolle & & nicolle.kord@txdot.gov & TxDOT \\
\hline Kragh, Brenda & & Brenda.Kragh@dot.gov & FOT/FHWA \\
\hline Krause, Pete & & pete.krause@txdot.gov & Texas DOT \\
\hline kuehn, david & & david.kuehn@dot.gov & fhwa \\
\hline $\begin{array}{l}\text { Kunsevich, } \\
\text { Alexandra }\end{array}$ & & alexndra.k@gmail.com & $\begin{array}{l}\text { Sequoia Mental Health } \\
\text { Services }\end{array}$ \\
\hline Kurt, Lee & 2319414300 & lee@traversetrails.org & TART Trails Inc. \\
\hline Levinger, David & & dlevinger@gmail.com & $\begin{array}{l}\text { Mobility Education } \\
\text { Foundation }\end{array}$ \\
\hline Lewis, Leonard & $\begin{array}{l}\text { (703) 259- } \\
1764\end{array}$ & Leonard.Lewis@VDOT.Virginia.gov & $\begin{array}{l}\text { Virginia Department of } \\
\text { Transportation }\end{array}$ \\
\hline Lissade, Herby & $916-417-6994$ & herby.lissade@dot.ca.gov & $\begin{array}{l}\text { California Department of } \\
\text { Transportation }\end{array}$ \\
\hline $\begin{array}{l}\text { Litzenberger, } \\
\text { John }\end{array}$ & $206515-3648$ & LITZENJ@WSDOT.WA.GOV & Washington State Ferries \\
\hline Loetz, Wesley & $303-757-9857$ & wesley.loetz@state.co.us & CDOT \\
\hline $\begin{array}{l}\text { Loveless, } \\
\text { Shirley }\end{array}$ & $610-566-1133$ & SML23@cornell.edu & Coleshill Associates LLC \\
\hline Luckey, Kara & & kara.luckey@gmail.com & $\begin{array}{l}\text { University of Colorado } \\
\text { Denver }\end{array}$ \\
\hline Lynch, Keith & $717-221-4545$ & keith.lynch@dot.gov & FHWA PA Division \\
\hline Macek, Ian & & maceki@wsdot.wa.gov & WSDOT \\
\hline Macon, Tameka & & Tameka.Macon@dot.gov & FHWA HQ \\
\hline macy, dennis & $405-254-3324$ & dennis.macy@dot.gov & fhwa/oklahoma \\
\hline
\end{tabular}

\begin{tabular}{|l|l|l|l|}
\hline $\begin{array}{l}\text { Malamud-Roam, } \\
\text { Frances }\end{array}$ & $\begin{array}{l}510-286- \\
7185\end{array}$ & $\begin{array}{l}\text { frances_malamud- } \\
\text { roam@dot.ca.gov }\end{array}$ & CALTRANS \\
\hline Maley, Barbara & 214.224 .2175 & barbara.maley@dot.gov & $\begin{array}{l}\text { US DOT / FHWA / Texas } \\
\text { Division }\end{array}$ \\
\hline $\begin{array}{l}\text { McClatchey, } \\
\text { Charles }\end{array}$ & & cemcclatchey@azdot.gov & $\begin{array}{l}\text { Arizona Department Of } \\
\text { Transportation }\end{array}$ \\
\hline McGee, Barbara & $\begin{array}{l}410-545- \\
8042\end{array}$ & bmcgee@sha.state.md.us & Safe Routes to School \\
\hline Miller, Heidi & & hmiller@northamptoncounty.org & $\begin{array}{l}\text { Northampton County } \\
\text { DCED }\end{array}$ \\
\hline
\end{tabular}




\begin{tabular}{|l|l|l|l|}
\hline Mitchell, Khan & & khan.mitchell@dot.gov & FHWA, PA Division \\
\hline Morris, Anne & $803429-7849$ & anne@anne-morris.com & $\begin{array}{l}\text { Anne morris and } \\
\text { Associates, LLC }\end{array}$ \\
\hline Morris, Michael & & michael.morris@dot.gov & FHWA-CA \\
\hline $\begin{array}{l}\text { Mrotek } \\
\text { Glenzinski, Jill }\end{array}$ & & jill.mrotekglenzinski@dot.wi.gov & WisDOT \\
\hline Muirhead, Kristel & $\begin{array}{l}541-322- \\
2994\end{array}$ & kmuirhead@bendoregon.gov & $\begin{array}{l}\text { City of Bend Police } \\
\text { Department }\end{array}$ \\
\hline Murphy, Veronica & & veronica.murphy@dot.state.nj.us & $\begin{array}{l}\text { NJ Department of } \\
\text { Transportation }\end{array}$ \\
\hline Nevins, Brian & & brian.nevins@dot.gov & FHWA \\
\hline O'Neill, Bryan & & Bryan.J.O'Neill@hud.gov & HUD \\
\hline Olu, Janice & $\begin{array}{l}202-402- \\
4587\end{array}$ & Janice.P.Olu@hud.gov & Department of HUD \\
\hline Osadczuk, Janice & & janice.osadczuk@dot.gov & FHWA \\
\hline Otero, Victor & & victor.otero@dot.gov & FHWA \\
\hline Padron, Jorge & 9547774320 & gaspar.padron@dot.state.fl.us & $\begin{array}{l}\text { Florida department of } \\
\text { Transportation }\end{array}$ \\
\hline Patterson, Gerius & & Gerius.Patterson@dot.gov & DFS-PMIT \\
\hline pearson, clifford & 2023669488 & clifford.pearson@dot.gov & fhwa \\
\hline Perkins, Dawn & $\begin{array}{l}573-638- \\
2626\end{array}$ & dawn.perkins@dot.gov & FHWA - MO Division \\
\hline
\end{tabular}

\begin{tabular}{|l|l|l|l|}
\hline $\begin{array}{l}\text { Petersen, } \\
\text { Jodi }\end{array}$ & Jodi.Petersen@dot.gov & FHWA \\
\hline Poe, Carson & $\begin{array}{l}617-494- \\
2765\end{array}$ & carson.poe@dot.gov & USDOT Volpe Center \\
\hline Pound, Bob & 7155814298 & rtpound@gmail.com & Weston Fire Dept \\
\hline Prewitt, Jeff & $\begin{array}{l}904-256- \\
2256\end{array}$ & jeffrey.prewitt@rsandh.com & RS\&H \\
\hline Randall, Lisa & $\begin{array}{l}320-963- \\
3209\end{array}$ & lisa.randall@dot.gov & FHWA \\
\hline $\begin{array}{l}\text { Rees, } \\
\text { Christine }\end{array}$ & 3037579836 & christine.rees@dot.state.co.us & $\begin{array}{l}\text { Colorado Department of } \\
\text { Transportation }\end{array}$ \\
\hline $\begin{array}{l}\text { Rehnborg, } \\
\text { Wayne }\end{array}$ & $\begin{array}{l}512-832- \\
7019\end{array}$ & wayne.rehnborg@txdot.gov & TxDOT - Austin District \\
\hline $\begin{array}{l}\text { Reynolds, } \\
\text { Ariadne }\end{array}$ & $\begin{array}{l}510 \text { 286- } \\
0342\end{array}$ & areynolds@scc.ca.gov & $\begin{array}{l}\text { California State Coastal } \\
\text { Conservancy }\end{array}$ \\
\hline $\begin{array}{l}\text { Robinette, } \\
\text { Jeff }\end{array}$ & $\begin{array}{l}304-347- \\
5931\end{array}$ & jeffrey.robinette@dot.gov & FHWA-West Virginia \\
\hline
\end{tabular}




\begin{tabular}{|l|l|l|l|}
\hline $\begin{array}{l}\text { Romero, } \\
\text { Elizabeth }\end{array}$ & elizabeth.romero@dot.gov & FHWA OK \\
\hline $\begin{array}{l}\text { Roper, } \\
\text { Rachel }\end{array}$ & & Rachel.LA.Roper@hawaii.gov & Hawaii DOT \\
\hline $\begin{array}{l}\text { Ross, } \\
\text { Vanessa }\end{array}$ & vanessa.ross@dot.gov & FHWA \\
\hline Rowe, Tim & $\begin{array}{l}775-888- \\
7357\end{array}$ & trowe@dot.state.nv.us & Nevada DOT \\
\hline $\begin{array}{l}\text { Sanders, } \\
\text { Michael }\end{array}$ & & MSanders@azdot.gov & Arizona DOT \\
\hline $\begin{array}{l}\text { Santiago, } \\
\text { Jessica }\end{array}$ & & jessica.a.santiago@odot.state.or.us & Oregon Dept. of Transportatino \\
\hline $\begin{array}{l}\text { Schneider, } \\
\text { Tom }\end{array}$ & toms12@gmail.com & Safe Passages Project \\
\hline $\begin{array}{l}\text { Shapley, } \\
\text { Darren }\end{array}$ & $\begin{array}{l}206-515- \\
3974\end{array}$ & shapled@wsdot.wa.gov & $\begin{array}{l}\text { Washington State Dept of } \\
\text { Transportation/Ferries Division }\end{array}$ \\
\hline $\begin{array}{l}\text { Smith, } \\
\text { Debbie }\end{array}$ & $\begin{array}{l}206-515- \\
3442\end{array}$ & dsmith@wsdot.wa.gov & Washington State Ferries \\
\hline $\begin{array}{l}\text { Smith, } \\
\text { Mamie }\end{array}$ & mamie.smith-fisher@dot.gov & FTA \\
\hline $\begin{array}{l}\text { Stafford, } \\
\text { Ryan }\end{array}$ & staffor@wsdot.wa.gov & WSDOT Area 1 Tacoma \\
\hline
\end{tabular}

\begin{tabular}{|l|l|l|l|}
\hline Stenkamp, Ken & $\begin{array}{l}541-312- \\
7943\end{array}$ & kstenkamp@bendoregon.gov & Bend Police Department \\
\hline Streisfeld, Lisa & $\begin{array}{l}719-227- \\
3248\end{array}$ & lisa.streisfeld@state.co.us & $\begin{array}{l}\text { Colorado Department of } \\
\text { Transportation }\end{array}$ \\
\hline $\begin{array}{l}\text { Suciu Smith, } \\
\text { Deborah }\end{array}$ & $\begin{array}{l}717-221- \\
3785\end{array}$ & deborah.suciu.smith@dot.gov & FHWA \\
\hline Swartz, Jon & $\begin{array}{l}406-444- \\
6158\end{array}$ & Joswartz@mt.gov & Montana DOT \\
\hline Sweeney, Mark & & sweeneym@michigan.gov & $\begin{array}{l}\text { State of Michigan - } \\
\text { MDOT }\end{array}$ \\
\hline sykes, randolph & 8085862309 & randolph.sykes@oahumpo.org & $\begin{array}{l}\text { oahu metropolitan } \\
\text { planning organization }\end{array}$ \\
\hline $\begin{array}{l}\text { Szwajkowski, } \\
\text { Kristin }\end{array}$ & & kszwajkowski@milligancpa.com & $\begin{array}{l}\text { Milligan and Company, } \\
\text { LLC }\end{array}$ \\
\hline $\begin{array}{l}\text { Talbert-Jackson, } \\
\text { Sandy }\end{array}$ & $\begin{array}{l}410-962- \\
0116\end{array}$ & sandy.talbert-jackson@dot.gov & FHWA RC \\
\hline Telfair, Brian & & brian.telfair@dot.gov & FHWA-FL \\
\hline Urban, Melinda & & melinda.urban@dot.gov & FHWA-CO \\
\hline
\end{tabular}




\begin{tabular}{|l|l|l|l|}
\hline $\begin{array}{l}\text { Vaughn-Fair, } \\
\text { Sharon }\end{array}$ & $\begin{array}{l}410-962- \\
2544\end{array}$ & Sharon.Vaughn-Fair@dot.gov & FHWA - HCC \\
\hline Venables, Ann & $\begin{array}{l}813-675- \\
6725\end{array}$ & ann.venables@urs.com & URS Corporation \\
\hline Vogt, Shawn & & vogts@wsdot.wa.gov & Washington State Ferries \\
\hline Vrana, Tammy & $\begin{array}{l}727-415- \\
1200\end{array}$ & tvrana@vciplanning.com & Vrana Consulting, Inc. \\
\hline Wade, Kelly & & kelly.wade@dot.gov & FHWA \\
\hline Wilhoit, James & 4254307319 & jwilhoit@rentonwa.gov & City of Renton \\
\hline Wilkinson, Cory & $\begin{array}{l}850-432- \\
6800\end{array}$ & cory.wilkinson@hdrinc.com & HDR Engineering \\
\hline Williams, Stan & 931.645 .7448 & stanwilliams@cityofclarksville.com & Clarksville MPO \\
\hline Wisth, Michael & 5416825540 & michael.c.wisth@ci.eugene.or.us & City of Eugene, Oregon \\
\hline Young, Marlene & $\begin{array}{l}808587- \\
2135\end{array}$ & marlene.q.young@hawaii.gov & $\begin{array}{l}\text { Dept. of Transportation } \\
\text { State of Hawaii }\end{array}$ \\
\hline
\end{tabular}

\begin{tabular}{|l|l|l|l|}
\hline Zandler, John & 602-463-0398 & jzandler@azdot.gov & Arizona Department of Transportation \\
\hline
\end{tabular} 


\section{APPENDIX B}

\begin{tabular}{|c|c|c|c|c|}
\hline \multicolumn{5}{|c|}{$\begin{array}{l}m \square \square \square \\
\square \square\end{array}$} \\
\hline $\begin{array}{l}\text { Wed. AM } \\
\text { Earty Eird }\end{array}$ & $\begin{array}{l}1: 30 \mathrm{~mm} \\
11: 5 \mathrm{~mm}\end{array}$ & \multicolumn{3}{|c|}{ Historic Columbia River Highway Reconnection Project Tour } \\
\hline Session & Time & $\begin{array}{c}\text { Session } 1 \\
\text { Mountsinview Room }\end{array}$ & $\begin{array}{l}\text { Session } 2 \\
\text { Riverview Room }\end{array}$ & $\begin{array}{c}\text { Session } 3 \\
\text { Shoreline Room }\end{array}$ \\
\hline \multicolumn{5}{|c|}{ Wednesday, October 3} \\
\hline 1 & $200 \mathrm{pm}-300$ & 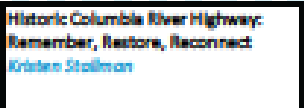 & 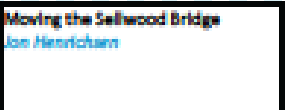 & 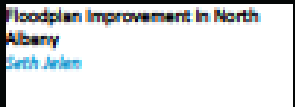 \\
\hline 2 & pmen-4:90 & 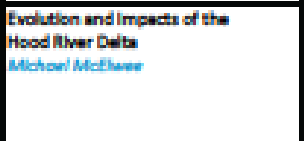 & 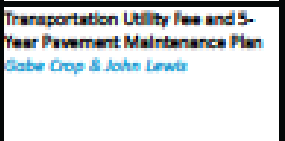 & 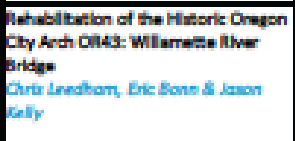 \\
\hline \multicolumn{5}{|c|}{ Thursday, October 4} \\
\hline 3 & $\begin{array}{l}2.00 \mathrm{sm}- \\
10000 \mathrm{~mm}\end{array}$ & 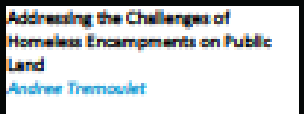 & 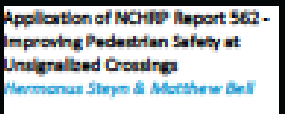 & 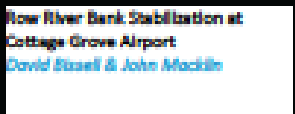 \\
\hline 4 & $10: 30 \mathrm{Am}-$ & $\begin{array}{l}\text { Rdaptive Managemant } \\
\text { Caver Murdock }\end{array}$ & 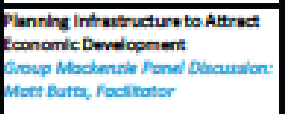 & 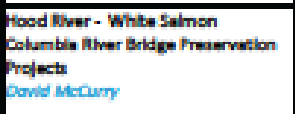 \\
\hline 5 & $200 \mathrm{pm}-9.00$ & 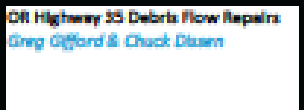 & $\begin{array}{l}\text { lock Solld Acrountability } \\
\text { Woe Cambe }\end{array}$ & 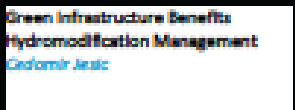 \\
\hline 6 & $930 p m-430$ & 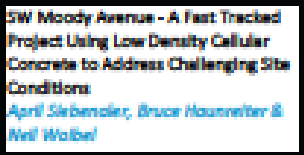 & 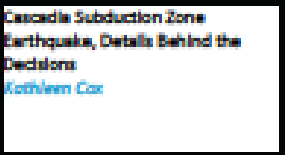 & 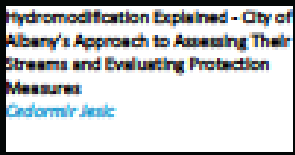 \\
\hline \multicolumn{5}{|c|}{ Pridey, October 3} \\
\hline 7 & $\begin{array}{l}p=30 \mathrm{~m}- \\
10-30=\mathrm{m}\end{array}$ & 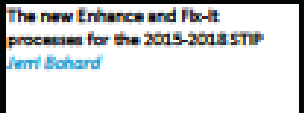 & $\begin{array}{l}\text { Lablik Worka on an ithad } \\
\text { ferry Kines }\end{array}$ & 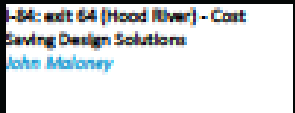 \\
\hline 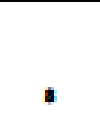 & $\begin{array}{l}11.00 \mathrm{~mm}- \\
12: 00 \mathrm{pm}\end{array}$ & 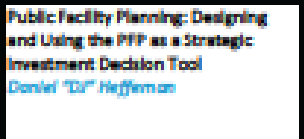 & 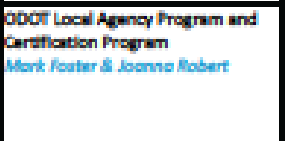 & 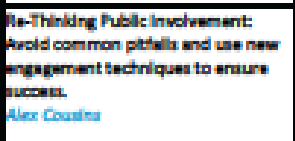 \\
\hline
\end{tabular}




\section{APPENDIX C}

\section{Hiding in Plain Sight: The Baldock Restoration Project}

On a winter night in 2010, 109 men, women and children slept at the Baldock Rest Area on I-5 south of Wilsonville, Oregon. Approximately one third of them had set up camp in back parking lots, away from the stream of visitors who stopped at the expansive rest area to take a break from driving. They were the chronically homeless, the self-named Baldockeans, the people whose lives revolved around the community at the Baldock. Some experienced physical or mental disabilities or addictions. The Baldock was their home, their refuge, their community.

The other two-thirds slept near parking area lights, where they felt safest. They were the "shadow people," the transitionally homeless. Their lives had hit a bad patch-the loss of a job, major debt from medical costs, divorce, domestic violence-and they had found themselves without sufficient income to stay in their homes. They did not identify as being part of the alternative world of the homeless; instead, their goal was to remain part of traditional society and regain their former status. During the day, they hid their homeless state, leaving the Baldock to work or spend time in libraries and other public places.

The long-term resident population had formed a complex, self-regulating community, with shared meals, organized shopping expeditions and delineated roles and responsibilities. One man had called the Baldcok home for 17 years, and St. Vincent de Paul, a social services agency, had provided weekly hot meals there for several years.

Some of the features that made the Baldock an attractive area for visitors also made it attractive to people without a permanent place to live. Hot and cold running water, toilets, picnic tables, water fountains, shady groves of trees and plenty of space were important amenities to people with only a vehicle, tent or camper as a home. It also provided privacy, with three parking areas on each side of the highway. For some, the steady stream of visitors provided a remunerative panhandling opportunity, and a few may have engaged in a grey market in prostitution or drugs. Others travelled to work from the Baldock. The rest area is just 14 miles south of Portland, with its urban services, and even closer to Canby. A truck stop a few miles to the south had showers, laundry facilities, a small market, a gas station and a restaurant. In short, the combination of amenities, relative privacy and location made it an attractive place to live for those with vehicles but no traditional homes.

That winter, the lives of the people sleeping at the Baldock were about to change. On January 1, Oregon Travel Experience (called Oregon Travel Information Council at the time) had assumed management responsibilities five rest areas in the state, including Baldock. In anticipation of this new role, in the fall OTE had organized a local business and public sector advisory committee to develop a vision for the 
Baldock Rest Area, and the group had expressed concerns about panhandling and other problems associated with the homeless community. On January 1, OTE was faced with the delicate decision of how to proceed.

OTE Executive Director Cheryl Gribskov chose hot chocolate.

\section{Homelessness and Transportation Agencies}

Although the homeless community at the Baldock Rest Area was unusual in its duration and sophistication, homeless encampments or urban campgrounds commonly occur on public land. In a national survey of state transportation agencies conducted by Bassett, Tremoulet and Moe in 2011, 70\% of respondents (representing 25 U.S. states and British Columbia) said that they encountered homeless encampments as part of their routine work. Any major public land owner with conveniently-located sites with some measure of privacy and shelter is a likely candidate for experiencing challenges with homeless individuals.

Upon learning of these research results, Emily Badger, a writer for The Atlantic Cities, commented, "This means that public agencies better equipped to run trains or pave highways must often act as the first responders to homelessness. It's a sad commentary on how we handle these populations-in a society that doesn't treat access to shelter as a right-that the task falls to the front-line employees of transportation agencies untrained to do anything like this."

\section{Hot Chocolate}

OTE was not the first agency that had attempted to deal with the homeless encampment at the Baldock Rest Area. Round-the-clock stays were against rest area rules, and Oregon Department of Transportation (ODOT) had, upon several occasions, called Oregon State Police to clear the area. However, neither the state police nor ODOT had sufficient resources to remain at the rest area on an ongoing basis, and thus the Baldockeans gradually returned. This cycle was repeated several times, creating a culture of distrust between the residents and state police.

Instead of leading with an enforcement-only approach, OTE decided that the agency needed to have a better understanding of the situation before proceeding. On New Year's Day 2010, Executive Director Gribskov and a community volunteer showed up at the rest area with hot chocolate to greet the residents, introduce themselves and listen to their concerns. Gribskov quickly realized that her agency alone could not solve the complex social, economic and political challenges underlying the presence of the Baldock community, so she sought help. She enlisted not just ODOT and Oregon State Police, but also state, county and local social service agencies, homeless advocates, local law enforcement, community leaders, and the county district attorney's office.

At fortuitously-timed Problem-Oriented Policing workshop sponsored by the Clackamas County District Attorney's Office, the basic strategy took shape in a committee comprised of social service and community justice representatives. The strategy involved intensive outreach and one-on-one assistance 
to provide opportunities to make changes that would enable them to move on from the Baldock to better living conditions. This was the "pull." It also involved changing the conditions that enabled Baldockeans to stay where they were. This "push" included changing the rest area rules and developing new methods of enforcement.

The period of transition would have to be a carefully-orchestrated ballet of pushing and pulling, with the professional partners presenting a humane but united front. The strategy came together in February 2010; the partners set a goal of clearing the rest area and beginning a higher level of enforcement on May 1, before the seasonal influx of new residents. It was a tall order, and initially there were no extra resources available to make it happen.

What made it happen was the personal commitment of the key partners involved: Ronell Warner of the Canby Center; Bill Stewart of the Clackamas County District Attorney's Office; Fred Testa and Dan Swift of Oregon State Police; Liz Bartell and Linda Fisher of Clackamas County Social Services; Karla Keller of Oregon Department of Transportation; Mary Carroll of Oregon Housing and Community Services; Amy Cleary and Cyndy Heisler of Clackamas County Domestic Resources Center and Cheryl Gribskov of Oregon Travel Experience. Eventually, a small amount of one-time-only funding - funds not available today- was found from state and county sources for enhanced case management, and local non-profits and donors chipped with donations of cash and supplies.

\section{Implementing a Push-Pull Approach}

On the pull side, social service agencies met with residents willing to accept assistance. They helped each person imagine and build a path to what they wanted for themselves. This could involve addressing old debts, obtaining a new social security card and identity papers to replace lost ones, taking responsibility for one's behavior to reunite with family, finding and accepting steady employment (no matter how hard), reinstating a commercial driver's license, enrolling in an in-patient substance abuse program, or any number of things. While the additional funds covered the costs of one-on-one case management, the pull partners creatively managed existing resources-classes, assessments-to bring services to the residents and tailor them to their needs.

The push-side partners developed new rules and enforcement procedures to dislodge the long-term community and ensure that a new one did not take its place. They tightened up Oregon Administrative Rules that governed behavior in rest areas and made failure to comply a Class B violation. The Clackamas County Community Court was poised to take on criminal cases if they arose and divert offenders to rehabilitative services as an alternative to serving jail time, if warranted. OTE made plans to refurbish the rest area and staff it with both a site team and volunteers to promote its use as a visitor resource. They also allocated funds to pay for enhanced police patrols after the enforcement date of May 1 and through the rest of the summer to discourage visitors from staying more than the allowed 12 hours during a 24-hour period.

The professional partners soon discovered that implementing a push-pull approach also required forging new levels of trust among themselves-social service agencies and law enforcement do not always see eye-to-eye on matters involving both their professions-and with the Baldockeans, who, 
until this point, had had little reason to believe that anyone cared. The professionals learned to trust each other's judgment and be flexible about enforcing the rules. They came to have each other's back, to be attentive to each other's safety and to show up if needed. For the Baldockeans, trust meant believing that these people were sincere about both the opportunities and permanent change at the Baldock. They began to believe that this time was different.

\section{Moving Day and Beyond}

Moving day, April $30^{\text {th }}, 2010$, was quite an event. The preceding 48 hours had involved a flurry of activity, with volunteer and paid mechanics working to get old vehicles road-worthy and the remaining Baldockeans packing their possessions. For those who had no other place to go immediately, the county had arranged temporary camping at a nearby state campground. Beyond that, they would do "in-andout" at the Baldock, staying no more than 12 hours at a time, until a more permanent solution was found.

Moving day was not picture-perfect, but it was successful. A state trooper who had not been involved with the project showed up unexpectedly and began ticketing the Baldockeans. Chaos ensued, and trust built over months of hard work was nearly destroyed, until the situation was sorted out by other state police who had been involved.

In the short term, half of the 20 people who had accepted county help found other places to camp, $30 \%$ did in-and-out at the Baldock, 10\% found permanent housing, 5\% went into detox and treatment, and $5 \%$ found other solutions. Approximately sixteen months later, half of them were in permanent housing and $15 \%$ were in transitional housing, waiting for a permanent spot to open up. Only $35 \%$ were in unstable living conditions. Given the circumstances, a long-term housing retention rate of $65 \%$ for this population is considered to be very good.

The Baldock is now a lovely, well-maintained visitor resource. The restored Grove of the States provides a walking path for those needing to stretch their legs. The back area sports a new solar array. While most who stop are there for just a short while, parking is also available for up to 12 hours a day to truck drivers and others (including former Baldockeans) who need a place to stop and sleep. There is no evidence of the long-standing community that once lived there.

\section{Learning from the Baldock}

The economic, social and political choices made as a nation over the last decades has ensured that our country will have an ongoing population of homeless individuals. Local housing and social services networks are, in most cases, struggling to keep up with the demand for services. Thus, homelessness is a messy, complicated societal problem with many spill-over effects, some of which are likely to continue to affect the maintenance and operations of our shared public land.

Perhaps there is an opportunity for those with the land and those with the services to sit down together with representatives or advocates of people without permanent housing to develop new approaches 
that accommodate the ongoing and permanent reality of homelessness in our communities today. The Baldock Restoration Project is one such example of that occurring. The solution reached was humane displacement.

In some cases, more long-term arrangements have been reached. Dignity Village, a self-managed homeless community, has a lease with the City of Portland for Sunderland Yard near the Portland airport. Over the last ten years, tents have been slowly replaced with small structures which must meet basic building codes for camping structures. Dignity Village is guided by a set of democratically-created rules, including no drugs, alcohol, disruptive behavior or children (for the children's safety). Residents must participate in weekly village meetings and contribute time and labor to maintaining the community.

In Eugene and nearby communities, St. Vincent de Paul manages an overnight parking program for homeless people with vehicles. They work with faith communities, non-profits, local governments and businesses that volunteer sites. St. Vincent de Paul provides garbage disposal and portable rest rooms. In 2011, the program assisted 81 individual adults and 27 families with 41 children.

In King County, Washington, the self-managed Tent City 4 rotates from one location to the next every ninety days, so that no one community absorbs the impact permanently. It is associated with the nonprofit SHARE/WHEEL. Currently, Tent City 4 is located in a church parking lot across from the police station in the prosperous town of Kirkland. Their self-imposed rules require that they be respectful neighbors, as they are often located in residential neighborhoods. Loitering outside the camp, parking nearby and loud noise are prohibited, as well as drugs and alcohol. They work with law enforcement to screen new community members.

Perhaps the ultimate challenge for collaboration was issued by a Federal Highway Administration official from the Midwest. At a January 2012 national transportation conference, where a case study of the Baldock Restoration Project was presented, he posed this question: What if transportation engineers started designing facilities such as overpasses or bridges to accommodate the needs of homeless people instead of chasing them away? Perhaps the sites could be maintained by social service agencies or selfmanaged communities. That solution would certainly represent a new kind of thinking about how to respond to the issue and require a new level of collaboration and trust among public agencies and the people whom they serve.

There is little question that living in a car, camper or tent in make-shift accommodations is far from the perfect long-term solution to the nation's intransigent problem of homelessness. But it may be a practical and humane one that is hiding in plain sight.

Word Count: 2,423 words

\section{Resources}

- A Case Study of the Baldock Restoration Project http://otrec.us/project/477 
- Homeless Encampments on Public Right-of-Way: A Planning and Best Practices Guide http://otrec.us/project/477

Andrée Tremoulet is a research associate for the Center for Urban Studies at Portland State and owner of Commonworks Consulting.

Ellen M. Bassett is an Associate Professor at the Department of Urban and Environmental Planning at the University of Virginia.

Allison Moe...[to be provided]

Research on the Baldock Restoration Project was funded by Oregon Department of Transportation and Oregon Transportation Research and Education Consortium. Views expressed in the article are the authors' own. 


\section{APPENDIX D}

\section{Response to 2013 Call for Transportation and Environmental Research Ideas \\ Final Submitted June 17, 2013}

Research Idea Title: Guidebook on Streamlining Delivery and Maintenance of Projects Involving Homeless Populations

Focus Areas: [from drop-down list] Streamlining Project Delivery

Cost: $\$ 250,000$

Time Frame: 2 years

Research Idea Scope: Please enter a brief summary of your research idea and objectives. If known, please include anticipated tasks required to complete the research project.

State DOTs, like many other agencies during this economic downturn, have struggled to provide basic services. At the same time, the housing market has imploded and the number of returning veterans with mental and psychological disorders has exploded. The combination of these two events has resulted in a noticeable increase in the number of homeless individuals and families with few economic resources no place to go for shelter. In many instances, these individuals and families have sought refuge in their cars at rest areas, under bridges, in spoils areas, and in other locations within public right-of-way. Efforts to evict these individuals and families have sometimes resulted in maintenance crews, local police, and highway patrol personnel being accosted, and some have become public relations nightmares for DOTs. DOTs have been sued over the disposal of the possessions and debris left at homeless camps. As a safety issue, it is the kind of problem that may be suitable for the Every Day Counts initiative for accelerating information, once solutions have been developed.

In most cases, DOTs have been poorly prepared to deal with this increasing problem and only focused on short-term removal of homeless individuals from the rights-of-way. In other cases, DOTs have collaborated with homeless services providers, public agencies, medical professionals, and other nonprofit and for-profit entities in an attempt to address the myriad of problems these individuals have and deliver long-term solutions. Because many of the affected properties have some level of Federal funding, and because most of these individuals are low-income, they are considered "environmental justice" populations and addressed by Executive Order 12898, entitled Federal Actions to Address Environmental Justice in Minority Populations and Low Income Populations.

This executive order requires that all federal agencies "identif[y] and addr[ess], as appropriate, 
disproportionately high and adverse human health or environmental effects of its programs, policies and activities on minority populations and low-income populations." According to the US DOT'S environmental justice (EJ) website, the requirements apply to all phases of a transportation facility's life cycle, including project development (planning, development, environmental review under the National Environmental Policy Act (NEPA), preliminary design and final design engineering), construction (right- of-way and construction), and maintenance (operation and maintenance).

As very low income individuals, the homeless population is covered under EJ requirements, but they are typically overlooked in transportation research and decision-making. Estimates of the size of the homeless population in the US range from 633,782 individuals (US Department of Housing and Urban Development, 2012) to as high as 3.5 million people (National Law Center on Homelessness, 2007), depending on definitions and methodology. People without shelter seek refuge wherever they can. As one of the nation's largest public land owners in areas with urban services, transportation agencies find themselves face-to-face with the challenge of dealing with this complex population every day. These challenges occur in every phase of a transportation facility's life cycle.

Currently, there is little research available on homeless populations in the transportation literature. That which does exist predominantly focuses on the transit needs of homeless populations or the impact of homeless individuals on transit agencies. In terms of highway development, construction and maintenance, there are two individual case studies about how Florida DOT and Washington DOT addressed the concerns of homeless individuals in two major construction projects, US-301 in Sarasota, FL (Potier-Brown \& Pipkin, 2005) and the Alaskan Way Viaduct and Seawall Replacement Project in Seattle, WA (Kocher, K. \& Kucharsky, M. 2007). There is one individual case study about the relocation of a homeless community from a rest area in Oregon (Tremoulet \& Bassett, 2012). A new publication analyzes how transportation agencies nationally have addressed the maintenance challenge of homeless encampments on public right-of-way and proposes collaborative strategies to create longterm solutions (Tremoulet, Bassett and Moe, 2012).

The objective of this research is to address this gap in knowledge by developing a Guidebook that provides information on how to incorporate planning for homeless populations in all three principal phases the life cycle of a highway-related transportation facility: development, construction and maintenance. The Guidebook's aim would be to help streamline project delivery through providing a systematized framework for addressing challenges related to homeless populations. For the development phase, the Community Impact Assessment approach provides a workable framework; the proposed Guidebook will address the gap in knowledge about how to adapt this framework to address the complex issues that arise in using it to work with people lacking permanent homes. For example, to reach this population, different methods of outreach and assessment are required because they may have no permanent address. The Uniform Relocation Act, an important resource in addressing adverse impacts on individuals and families, may apply differently to this population, as they may not qualify as displaced individuals. The main phase will build upon the existing preliminary national study about homeless encampments on right-of-way (Tremoulet, Bassett and Moe, 2012). Products from this research could be disseminated through the Every Day Counts Initiative of FHWA, as it relates to 
safety.

The research may include the following tasks:

1. Review transportation literature for information about the intersection of highway development, construction and maintenance and homeless populations. Review existing literature about how other professions have addressed similar challenges related to the impacts of homeless or transient populations on facility development.

2. Work with a panel of transportation experts (e.g., EJ, civil rights, right-of-way, NEPA, URA) and others to scope the project, identify potential examples to study, and explore the application, requirements and limitations of existing transportation regulations and resources as they relate to this population. Include in the panel potential national allies who may have an interest in collaborating around the development of best practices, including those identified by the federal Interagency Task Force on Homelessness.

3. Develop case studies of successful highway-related projects involving homeless people. Collectively, the case studies should cover all the stages in a transportation facility's life cycle (development, construction and maintenance).

4. Research non-transportation resources that may be useful to transportation personnel in working with homeless populations.

5. Develop preliminary research findings based on case studies, best practices research, existing regulatory framework and available transportation and external resources. Formulate preliminary principles, approaches and resources relevant to each phase. Review with Expert Panel and refine.

6. Finalize a Guidebook that helps transportation personnel streamline project delivery (development and construction phases) and ongoing operations through consistently addressing issues related to homeless individuals.

\section{$\underline{\text { Urgency and Payoff }}$}

Recent events highlight a strong and growing interest in the emerging topic of EJ issues associated with homeless populations, especially among state Departments of Transportation. An online survey of transportation agency personnel from 24 states conducted by Portland State University in 2011 found that $76 \%$ of the states reported issues with homeless encampments or individuals on right-of-way. Their principal concerns were with the safety of their personnel, motorists, the public, and the homeless individuals themselves. This research found that other problems caused by this population included debris and hazardous materials, damage to public facilities, short-term displacement of intended uses and users, and potential community, public, media and governmental relations issues for state DOTs. However, the research found that few transportation agencies had examined the problem, developed strategies for addressing it, or provided training to staff.

A May 23, 2013, FHWA webinar entitled Strategies for Addressing the Challenges of Transient Populations on Transportation Facilities attracted an audience of 141 participants, including personnel from 16 FHWA divisions and 13 state DOTs, as well as local officials, social service agencies and others interested in partnering on potential solutions to the challenges. Approximately one quarter of those participating in the webinar poll expressed interest in two follow-up areas: research 
and guidance on how to address impacts of homeless populations on the design and construction of projects and more information on how to address maintenance issues.

In response to the growing interest in this emerging topic, the ADD 50 Environmental Justice in Transportation Committee has elected to submit this research idea. This committee has also proposed sponsoring a Cross-Cutting Session on Homeless Populations on Right-of-Way with the Maintenance and Operations Management Committee at the 2014 Transportation Research Board Annual Meeting. Idea suggested by-

Your name: Andree Tremoulet

Agency/organization: In behalf of ADD 50 Environmental Justice in Transportation Committee

Your e-mail: atrem@pdx.edu

Your telephone: $503-725-4075$ 



\section{GOTREC \\ AND EDUCATION CONSORTIUM}

P.O. Box 751

Portland, OR 97207

OTREC is dedicated to stimulating and conducting collaborative multi-disciplinary research on multi-modal surface transportation issues, educating a diverse array of current practitioners and future leaders in the transportation field, and encouraging implementation of relevant research results. 